\title{
Análisis de sensibilidad de la prueba de Student para una muestra
}

\author{
Sensitivity Analysis for Student-Test in one sample \\ Jorge Ortiz Pinilla \\ jorgeortiz@usantotomas.edu.co \\ Heivar Yesid Rodríguez Pinzón \\ heivarrodriguez@usantotomas.edu.co
}

\section{Resumen}

La estadística $t$ de Student no es monótona en función de la diferencia entre $\bar{x}$ y $\mu_{0}$, pues ésta se modifica mediante alteraciones en los datos que también afectan el valor de la varianza. En este trabajo se utiliza esta propiedad para analizar la estabilidad en las pruebas cuando se encuentran valores-p cercanos del nivel de significación dado por el investigador.

Palabras clave: prueba $t$, monotonía, estabilidad.

\begin{abstract}
Student's $t$ statistic is not a monotonic function of the difference between $\bar{x}$ and $\mu_{0}$, since it is modified by alterations in the data that also affect the value of the variance. In this paper the property is used to analyze the stability of the test when the p-value is close to the significance level.
\end{abstract}

Key words: $t$ Statistic, Monotonicity, Stability.

\section{Introducción}

En la aplicación de la prueba $t$, la decisión de rechazo de la hipótesis nula se toma con base en el valor-p, calculado con los datos de la muestra observada, o a partir de la posición del valor de la estadística fuera del intervalo conocido como la región de aceptación. Por lo general, los estudios que abordan el tema de la calidad de las pruebas se centran en el comportamiento de la función de potencia. En algunos, sólo se trata el caso particular correspondiente a $\mu_{X}=\mu_{0}$, considerando que la prueba se comporta bien si la proporción de rechazos de $H_{0}: \mu_{X}=\mu_{0}$ coincide

\footnotetext{
${ }^{a}$ Docente. Facultad de Estadística. Universidad Santo Tomás.

${ }^{\mathrm{b}}$ Docente. Facultad de Estadística. Universidad Santo Tomás.
} 
con el nivel de significación utilizado. Más detalles y referencias adicionales se encuentran en (Montilla 2010).

En ocasiones, el investigador desconoce en su aplicación específica que su decisión puede verse afectada por un simple cambio en la cantidad de dígitos de sus datos o por un cambio muy pequeño en uno solo de ellos. Se encuentran casos más complejos cuando se rechaza la hipótesis nula con una muestra cuyo promedio se encuentra a una distancia $d_{1}$ del valor de la media poblacional especificado en $H_{0}$ y una alteración del valor más extremo de la muestra lleva a no rechazar $H_{0}$ y el valor del promedio con los nuevos datos se encuentra a una mayor distancia $d_{2}$ del mismo valor poblacional mencionado.

En este trabajo proponemos la aplicación de un análisis de sensibilidad para los resultados de la prueba $t$ para una muestra y su presentación como parte del informe respectivo para que el investigador tenga un mejor conocimiento de las condiciones en las que toma sus decisiones.

\section{Notación y cambios en un dato}

Se supone que $X_{1}, X_{2}, \ldots, X_{n}$ es una muestra aleatoria de una distribución normal $N\left(\mu, \sigma^{2}\right)$ y que $x_{1}, x_{2}, \ldots, x_{n}$ es una realización. $\bar{X}$ y $S_{X}^{2}$ son las estadísticas conocidas como la media y la varianza muestrales y $\bar{x}$ y $s_{x}^{2}$ son los valores respectivos calculados con los datos obtenidos. Además, $x_{(1)}, x_{(2)}, \ldots, x_{(n)}$ representa el conjunto de valores ordenados de menor a mayor.

Si se establece la hipótesis $H_{0}: \mu=\mu_{0}$ versus alguna de las alternativas conocidas, se utiliza la estadística $T_{X}=\left(\bar{X}-\mu_{0}\right) /\left(S_{X} / \sqrt{n}\right)$, cuyo valor para la muestra observada se denota como $t_{x}$. Como de costumbre, para una probabilidad $\alpha, t_{n-1}(\alpha)$ es el percentil correspondiente de la distribución $t$ de Student con $n-1$ grados de libertad, pero si no hay lugar a confusión, se escribe solamente $t_{\alpha}$. Con la simetría de la distribución con respecto a cero se tiene que $t_{\alpha}=-t_{1-\alpha}$.

Se considera $d$, una constante que se utiliza para modificar aditivamente uno de los datos, por ejemplo, $x_{(i)}^{*}=x_{(i)}+d$, donde $i$ puede ser cualquiera de $1,2, \ldots, n$. El promedio y la varianza afectados por esa modificación son $\overline{x_{i}^{*}}$ y $s_{x_{i}^{*}}^{2}$ y se representa el valor correspondiente de la estadística de Student como $t_{x_{i}^{*}}$.

\section{Efecto sobre la media y la varianza}

Si el dato $x_{(i)}$ se reemplaza por $x_{(i)}^{*}=x_{(i)}+d$, siendo $d$ una constante, entonces:

$$
\begin{aligned}
\overline{x_{i}^{*}} & =\frac{1}{n} \sum_{j=1, j \neq i}^{n} x_{(j)}+\frac{1}{n} x_{(i)}^{*} \\
& =\bar{x}+\frac{d}{n}
\end{aligned}
$$

Comunicaciones en Estadística, diciembre 2011, Vol. 4, No. 2 
A partir de (11) se concluye que el cambio ocasionado en el promedio no depende de cuál fue el dato transformado.

Para la varianza, se tiene:

$$
s_{x_{i}^{*}}^{2}=\frac{1}{n-1} \sum_{j=1, j \neq i}^{n}\left(x_{(j)}-\overline{x_{i}^{*}}\right)^{2}+\frac{1}{n-1}\left(x_{(i)}^{*}-\overline{x_{i}^{*}}\right)^{2}
$$

Haciendo los reemplazos y los desarrollos convenientes, se llega a:

$$
s_{x_{i}^{*}}^{2}=s_{x}^{2}+2\left(\frac{x_{(i)}-\bar{x}}{n-1}\right) d+\frac{d^{2}}{n}
$$

A diferencia de lo encontrado para el promedio, en (2) se observa que el cambio ocasionado en la varianza depende del valor afectado. Además, si $x_{(i)}-\bar{x}$ y $d$ tienen el mismo signo, la nueva varianza toma valores mayores que cuando $x_{(i)}-\bar{x}$ y $d$ tienen signos contrarios.

\section{Efecto sobre el valor de la estadística $t_{x}$}

Los efectos del cambio generado en un dato sobre el promedio y sobre la varianza tienen consecuencias en el valor de la estadística de prueba. Con los resultados de la sección anterior, se tiene:

$$
\begin{aligned}
t_{x_{i}^{*}}^{2} & =\frac{\left(\overline{x_{i}^{*}}-\mu_{0}\right)^{2}}{s_{x_{i}^{*}}^{2}} n \\
& =\frac{\left(\bar{x}+\frac{d}{n}-\mu_{0}\right)^{2}}{s_{x}^{2}+2\left(\frac{x_{(i)}-\bar{x}}{n-1}\right) d+\frac{d^{2}}{n}} n \\
& =\frac{\left(\bar{x}-\mu_{0}\right)^{2}+2\left(\bar{x}-\mu_{0}\right) \frac{d}{n}+\frac{d^{2}}{n^{2}}}{s_{x}^{2}+2\left(\frac{x_{(i)}-\bar{x}}{n-1}\right) d+\frac{d^{2}}{n}} n \\
& =\frac{s_{x}^{2} t_{x}^{2}+A}{s_{x}^{2}+B}
\end{aligned}
$$

donde

y

$$
A=2\left(\bar{x}-\mu_{0}\right) d+\frac{d^{2}}{n}
$$

$$
B=2\left(\frac{x_{(i)}-\bar{x}}{n-1}\right) d+\frac{d^{2}}{n}
$$


Como consecuencia de (11) y (2), puede verse en (3) que el cambio generado por $d$ en el numerador no depende del dato $x_{(i)}$ afectado, pero sí el ocasionado en el denominador.

Con un mismo valor de $d$ se puede afectar a $t_{x^{*}}^{2}$ en mayor o menor medida dependiendo de la diferencia que presente el dato que se modifica con respecto al promedio. De esta manera, con un mismo valor de $d, t_{x^{*}}^{2}$ toma el valor más grande si se modifica $x_{(1)}$ cuando $d>0$ o $x_{(n)}$ cuando $d<0$, es decir, acercando uno de los extremos.

De manera análoga, si se busca el valor más pequeño de $t_{x^{*}}^{2}$ habiendo fijado $d$, se debe aplicar la modificación a $x_{(1)}$ si $d<0$ o a $x_{(n)}$ si $d>0$, es decir, alejando alguno de los extremos.

De manera similar se tiene la siguiente relación entre $t_{x^{*}}$ y $t_{x}$ :

$$
\begin{aligned}
t_{x_{i}^{*}} & =\frac{\bar{x}-\mu_{0}+\frac{d}{n}}{s_{x_{i}^{*}}} \sqrt{n} \\
& =\frac{s_{x} t_{x}+\frac{d}{\sqrt{n}}}{s_{x_{i}^{*}}} \\
& =\frac{s_{x} t_{x}+\frac{d}{\sqrt{n}}}{\sqrt{s_{x}^{2}+B}}
\end{aligned}
$$

El valor más extremo que puede tomar la estadística con esta transformación se encuentra derivando $t_{x_{i}^{*}}$ con respecto a $d$ e igualando a cero. Así, el máximo o el mínimo, segun el caso, se encuentra en:

$$
d=\frac{\left(\frac{x_{(i)}-\bar{x}}{n-1} t_{x}-\frac{1}{\sqrt{n}} s_{x}\right) s_{x}}{\frac{x_{(i)}-\bar{x}}{n-1} \frac{1}{\sqrt{n}}-\frac{s_{x} t_{x}}{n}}
$$

\section{Efecto sobre la decisión sobre $H_{0}$}

Fácilmente se demuestra que $\lim _{d \rightarrow-\infty} t_{x_{i}^{*}}^{2}=\lim _{d \rightarrow \infty} t_{x_{i}^{*}}^{2}=1$, independientemente de la muestra observada, de su tamaño, de $\alpha$ y de $\mu_{0}$. Esto implica que sin importar el resultado obtenido para $t_{x}$, se puede encontrar un valor para $d$ que lleve a que $t_{x_{i}^{*}}^{2}$ quede suficientemente cerca de 1 como para que la prueba realizada con los datos transformados no rechace $H_{0}$, sin importar tampoco la alternativa con la cual se contraste.

Como consecuencia, cuando una prueba realizada con una muestra observada $x_{1}, x_{2}, \ldots, x_{n}$ lleva a rechazar $H_{0}$, siempre es posible encontrar una constante 
$d$, que agregada a $x_{(i)}$ conduzca a no rechazar $H_{0}$ con la muestra transformada. Cuando no se rechaza $H_{0}$ con la muestra observada, no siempre es posible encontrar una constante $d$ que lleve a la decisión contraria.

Si $t_{\alpha}$ es el percentil $\alpha$ de la distribución $t$ con $n-1$ grados de libertad, entonces, se puede buscar $d$ tal que:

$$
t_{x_{i}^{*}}^{2}=t_{\alpha}^{2}
$$

es decir,

$$
\frac{s_{x}^{2} t_{x}^{2}+2\left(\bar{x}-\mu_{0}\right) d+\frac{d^{2}}{n}}{s_{x}^{2}+2\left(\frac{x_{(i)}-\bar{x}}{n-1}\right) d+\frac{d^{2}}{n}}=t_{\alpha}^{2}
$$

Multiplicando por el denominador de la izquierda y reagrupando los términos, se obtiene la siguiente ecuación de segundo grado en $d$ :

$$
\frac{t_{\alpha}^{2}-1}{n} d^{2}+2\left(\frac{x_{(i)}-\bar{x}}{n-1} t_{\alpha}^{2}-\left(\bar{x}-\mu_{0}\right)\right) d+s_{x}^{2}\left(t_{\alpha}^{2}-t_{x}^{2}\right)=0
$$

Renombrando como $a, b$ y $c$ los coeficientes respectivos de $d^{2}, d$ y el término independiente en (9), se encuentran las soluciones

$$
\begin{aligned}
& d_{1}=\frac{-b-\sqrt{b^{2}-4 a c}}{2 a} \\
& d_{2}=\frac{-b+\sqrt{b^{2}-4 a c}}{2 a}
\end{aligned}
$$

siempre y cuando se cumpla que $b^{2} \geq 4 a c$.

El planteamiento de la ecuación

$$
t_{x_{i}^{*}}=t_{\alpha}
$$

lleva a la misma ecuación de segundo grado (9).

Teniendo en cuenta que para los niveles de significación usuales $t_{\alpha}^{2}>1$, si se rechaza $H_{0}$ con la muestra observada, es porque $t_{x}^{2} \geq t_{\alpha}^{2}$ y se tiene garantía de encontrar un valor real para $d$ que hace cambiar la decisión sobre $H_{0}$, como se vio antes.

Si $t_{x}^{2} \geq t_{\alpha}^{2}$ y no se rechaza $H_{0}$, no siempre es posible encontrar valores para $d$ que hagan cambiar la decisión. Sin embargo, si $t_{x}^{2}<t_{\alpha}^{2}$, pero con una diferencia pequeña, de manera que $4 a c$ sea pequeño y si $x_{(i)}-\bar{x}$ y $\mu_{0}-\bar{x}$ tienen el mismo signo, para que $b$ tome un valor mayor que $4 a c$, entonces se encuentran soluciones para (9). Esto se facilita si se toma $i=n$ cuando $\mu_{0}>\bar{x}$ o $i=1$ cuando $\mu_{0}<\bar{x}$. Es claro que si el valor más extremo posible de $t_{x_{i}^{*}}$, calculado con $d$ dado en (7), es menos extremo que el punto crítico de la prueba, entonces no existe la posibilidad de cambiar la decisión de no rechazar $H_{0}$. 


\section{Indicador de estabilidad de la prueba}

Cuando no existen soluciones reales para $d$, la prueba se considera estable para este tipo de modificaciones, pues con ellas no habrá manera de cambiar la decisión. Si existen, la magnitud de $d$ puede interpretarse como el "intervalo de seguridad" donde $x_{(i)}$ puede moverse sin generar cambios en la decisión tomada con la muestra observada originalmente. En este sentido, la solución de menor valor absoluto es un indicador de la estabilidad de la prueba para el tipo de modificación planteado, siendo los valores pequeños los que corresponden a poca estabilidad.

Si el valor de la solución encontrada es muy pequeño, el escaso margen de movimiento indica que la decisión tomada es inestable y es bueno que el investigador lo sepa, no para que cambie su decisión, que ya no está en juego, pero sí para que tenga en cuenta este aspecto en sus interpretaciones.

La figura 11(a) ilustra una situación de inestabilidad para una prueba unilateral izquierda $\left(H_{0}: \mu_{X} \geq \mu_{0}\right.$ contra $\left.H_{1}: \mu<\mu_{0}\right)$. El valor de la estadística observada con los datos originales corresponde a la posición $d=0$, sin alterar ningún valor de la muestra y la prueba no rechaza la hipótesis nula.

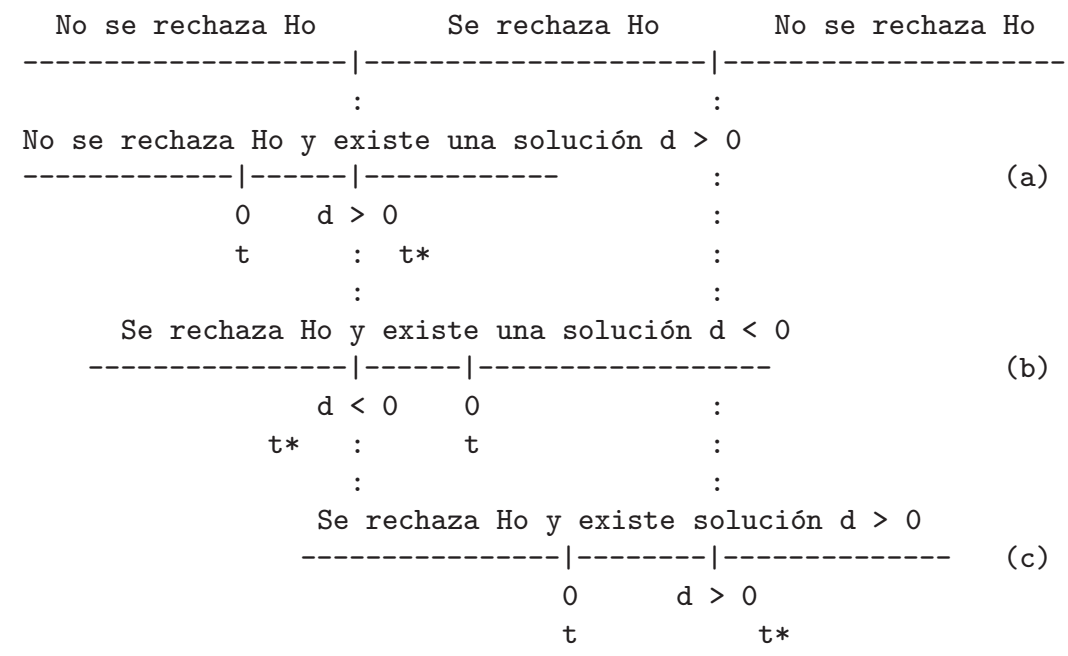

Figura 1: Situaciones inestables para la decisión sobre $H_{0}$.

Si, para el caso, existe una solución $d>0$, el promedio modificado por un cambio $d_{1}$ ligeramente mayor que esta solución es mayor que el observado, es decir, más cercano numéricamente del valor dado en $H_{0}$, pero con el cual se obtendría un valor $t^{*}$ conducente a rechazar esta hipótesis. 
En la figura 1(b) la posición de la estadística, correspondiente a $d=0$, está en la región de rechazo y se tiene una solución $d<0$. Si $x_{(i)}$ hubiera tomado el valor $x_{(i)}+d_{1}$, con $d_{1}<d$, no se hubiera rechazado $H_{0}$. Se tiene una incomodidad similar a la del caso anterior, en el sentido de encontrar ahora que un promedio numéricamente más lejano del especificado en $H_{0}$ llevaría a no rechazarla.

Las "incomodidades" mencionadas en los casos (a) y (b) se explican por el hecho de que la prueba $t$ no examina las diferencias numéricas entre $\bar{x}$ y $\mu_{0}$ en bruto, sino en unidades del error estándar, lo que se constituye en el patrón para evaluar su comportamiento probabilístico.

La figura 1(c) ilustra el caso en que con el valor obtenido de la estadistica se rechaza $H_{0} \mathrm{y}$ en el análisis de sensibilidad se encuentra una solución $d>0$, cuyo efecto hubiera sido acercar el promedio al dado en $H_{0}$. Así que si en lugar de $x_{(i)}$ se hubiera observado $x_{(i)}+d_{1}$, no se hubiera rechazado $H_{0}$.

Puede ser conveniente presentar tanto el valor bruto, que indica el tamaño de la alteración que sería necesario aplicar, como el valor en unidades de desviación estándar para tener idea de la magnitud de la modificación en función de la dispersión de los datos.

Con el siguiente código en $\mathrm{R}$ se encuentran las soluciones de la ecuación (91). Si no son soluciones reales, la función retorna el valor Inf para indicarlo.

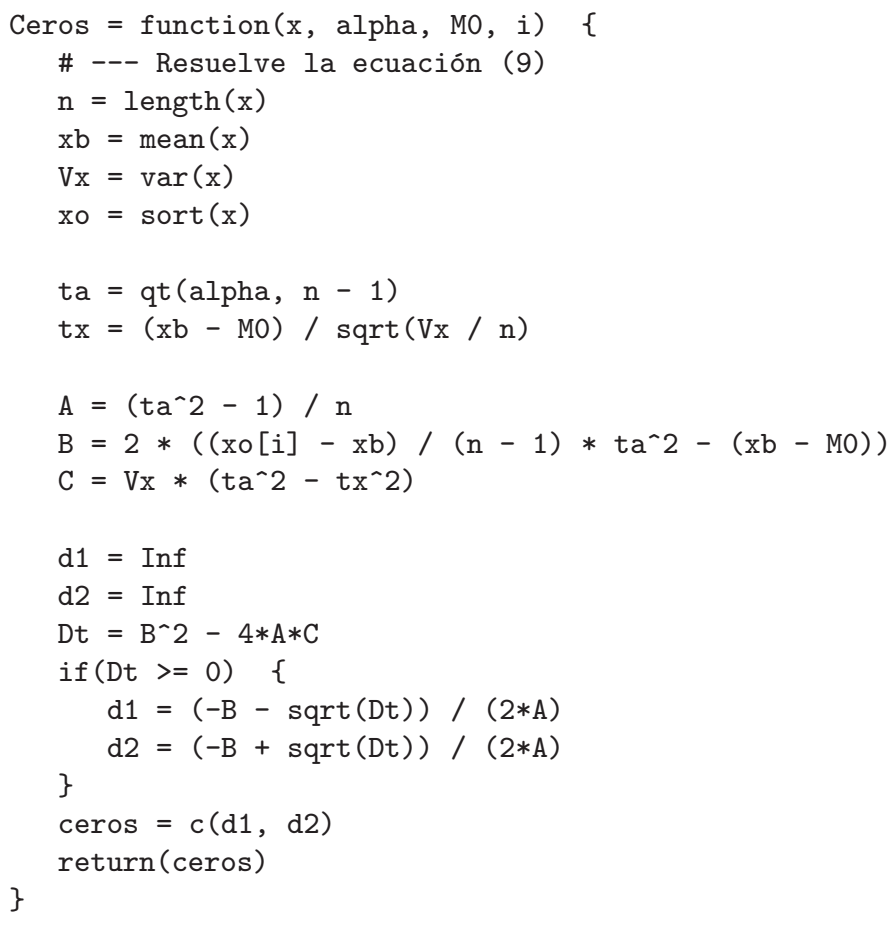


Ejemplo: Para probar $H_{0}: \mu_{X} \geq 10.5$ contra $H_{1}<10.5$, con $\alpha=0.05$ y con los siguientes datos: $4.9,6.4,7.0,7.9,8.6,9.5,9.5,9.6,10.0,10.3,10.6,11.8,12.0,12.0,12.3$, se tiene:

$\bar{x}=9.493333, s_{x}=2.205988, t_{x}=-1.7674, g l=14$, valor-p $=0.04947$.

Para $x_{(1)}$ se encuentran las constantes -0.785986 y 0.945172 que corresponden a -0.3562966 y 0.4284576 desviaciones estándar, respectivamente.

Cualquier valor tomado del intervalo $[-0.785986,0.945172]$ y que se agregara a $x_{(1)}$ generaría un valor de $t^{*}$ que llevaría a rechazar $H_{0}$ en favor de $H_{1}: \mu<10.5$, si se tomara $t^{*}$ para decidir. Fuera de este intervalo, no se genera rechazo de $H_{0}$.

Por ejemplo, si en lugar de $x_{(1)}=4.9$, se hubiera tenido $x_{(1)}=4.1$, los resultados hubieran sido: $\bar{x}=9.44 s_{x}=2.331094, t=-1.7611, g l=14$ y valor-p $=0.05002$ y si $x_{(1)}=5.9$, entonces: $\bar{x}=9.56, s_{x}=2.068056, t=-1.7604, g l=14$ y valor-p $=0.05008$.

Para $x_{(n)}$, el indicador de estabilidad es de 0.03192084 . Si en la muestra original se hubiera encontrado $x_{(n)}=12.7$ en lugar de $x_{(n)}=12.3$, la decisión hubiera cambiado.

\section{Conclusiones}

1. Cuando la ecuación (9) tiene soluciones reales, es posible encontrar constantes $d$ que al ser agregadas al dato $x_{(i)}$ de la muestra observada llevan a modificar la decisión tomada sobre $H_{0}$.

2. Cuando se rechaza la hipótesis nula, siempre es posible encontrar constantes $d$ que al ser agregadas a uno de los datos de la muestra observada acercan $\bar{x}$ de $\mu_{0}$ y al aplicar la prueba $t$ a los datos modificados ya no permiten rechazar $H_{0}$. La de menor valor absoluto se reconoce como indicador de estabilidad.

3. Cuando se rechaza la hipótesis nula, la ecuación (9) siempre tiene soluciones reales y, por lo tanto, se pueden encontrar constantes $d$ que al ser agregadas al dato $x_{(i)}$ de la muestra observada, alejan $\bar{x}$ de $\mu_{0} \mathrm{y}$, sin embargo, al aplicar la prueba $t$ a los datos modificados ya no se rechaza $H_{0}$.

4. En cualquier caso, a menor valor del indicador, menor será la estabilidad del resultado obtenido con la muestra observada.

5. Cuando la ecuación (9) no tiene soluciones reales, el resultado se considera absolutamente estable.

6. Una misma constante $d$ genera modificaciones más grandes en la estadística de prueba si se agrega a $x_{(1)}$ o a $x_{(n)}$.

Es importante no descontextualizar el análisis propuesto, que se aplica exclusivamente al resultado observado. No se pueden trasladar las conclusiones a la prueba 
$t$ de manera general. Lo estable o inestable es el resultado y no la prueba, pues lo que hemos llamado la muestra modificada no es ya una muestra aleatoria sino una muestra manipulada, en el contexto de análisis de sensibilidad, para examinar condiciones que pudieran afectar la estabilidad de las decisiones tomadas.

\section{Agradecimientos}

Los autores agradecen los valiosos comentarios hechos por el profesor Julio Da Motta Singer, durante el XX Simposio de Estadística.

Recibido: 10 de enero de 2011 Aceptado: 8 de septiembre de 2011

\section{Referencias}

Montilla, J., M. a. K. J. (2010), 'Robustez de las pruebas $T$ en comparación de medias, ante violación de supuestos de normalidad y homocedasticidad', Revista Ciencia e Ingeniería 31(2), 101-108.

R Development Core Team (2007), R: A Language and Environment for Statistical Computing, R Foundation for Statistical Computing, Vienna, Austria. ISBN 3-900051-07-0.

*http://www.R-project.org 